\title{
ON A COUNTEREXAMPLE TO A CONJECTURE BY BLACKADAR
}

\author{
ADAM P. W. SØRENSEN
}

\begin{abstract}
Blackadar conjectured that if we have a split short-exact sequence $0 \rightarrow I \rightarrow A \rightarrow \mathbb{C} \rightarrow 0$ where $I$ is semiprojective then $A$ must be semiprojective. Eilers and Katsura have found a counterexample to this conjecture. Presumably Blackadar asked that the extension be split to make it more likely that semiprojectivity of $I$ would imply semiprojectivity of $A$. But oddly enough, in all the counterexamples of Eilers and Katsura the quotient map from $A$ to $A / I \cong \mathbb{C}$ is split. We will show how to modify their examples to find a non-semiprojective $C^{*}$-algebra $B$ with a semiprojective ideal $J$ such that $B / J$ is the complex numbers and the quotient map does not split.
\end{abstract}

\section{INTRODUCTION}

Semiprojectivity is a lifting property for $C^{*}$-algebras. It was introduced in [1] in a successful attempt to transfer some of the power of shape theory for metric spaces to the world of $C^{*}$-algebras.

Definition 1. $A C^{*}$-algebra $A$ is semiprojective if whenever we have a $C^{*}$-algebra $B$ containing an increasing sequence of ideals $J_{1} \subseteq J_{2} \subseteq \cdots$, and $a *$-homomorphism $\phi: A \rightarrow B / \overline{\cup_{k} J_{k}}$, we can find an $n \in \mathbb{N}$ and $a$-homomorphism $\psi: A \rightarrow B / J_{n}$ such that

$$
\pi_{n, \infty} \circ \psi=\phi,
$$

where $\pi_{n, \infty}: B / J_{n} \rightarrow B / \overline{\cup_{k} J_{k}}$ is the natural quotient map.

Pictorially, $A$ is semiprojective if we can always fill in the dashed arrow in the following commutative diagram:

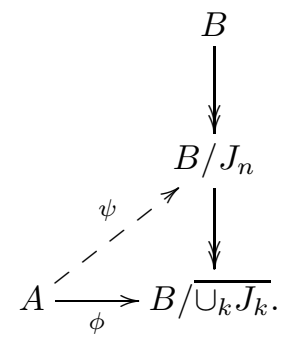

The book [10] is the canonical source for information about semiprojectivity. See also the more recent paper [2, the beginning of which has an expository nature.

Many of the main problems about semiprojectivity are concerned with the permanence properties of semiprojective $C^{*}$-algebras. In [1] Blackadar proves that the direct sum of two unital semiprojective $C^{*}$-algebras is again semiprojective, and that if $A$ is unital and semiprojective then $M_{n}(A)$ is also semiprojective. These results where later extended from unital algebras to $\sigma$-unital algebras, so in particular to all separable algebras, by Loring in [9]. The results are a little stronger, in fact we have for separable algebras that $A \oplus B$ is semiprojective if and only if

Date: June 19, 2018. 
both $A$ and $B$ are, and a separable unital algebra $D$ is semiprojective if and only if $M_{2}(D)$ is. It is still an open problem if a non-unital $A$ must be semiprojective whenever $M_{2}(A)$ is. It is true if $A$ is commutative, see [16. Corollary 6.9].

For a long time the following conjecture by Blackadar ([2, Conjecture 4.5]), which was first asked as a question by Loring in 10, was one of the main questions concerning the permanence properties of semiprojective $C^{*}$-algebras:

Conjecture 1 (Blackadar). Let

$$
0 \rightarrow A \rightarrow B \rightarrow \mathbb{C} \rightarrow 0
$$

be a split exact sequence of separable $C^{*}$-algebras. If $A$ is semiprojective then so is $B$.

An important partial result was obtained in [5, Theorem 6.2.1]. It was used in [5] to show that all the so called one-dimensional non-commutativ CW complexes are semiprojective. Enders (6] has proved a form of converse to Conjecture 1, namely that if $0 \rightarrow A \rightarrow B \rightarrow \mathbb{C} \rightarrow 0$ is an exact sequence of separable $C^{*}$-algebras with $B$ semiprojective then $A$ is semiprojective.

Recently Eilers and Katsura (4 ) have found a counterexample to Conjecture 1 .

Theorem 1 (Eilers-Katsura). There exists a split short exact sequence

$$
0 \rightarrow A \rightarrow B \rightarrow \mathbb{C} \rightarrow 0
$$

where $A$ is semiprojective but $B$ is not.

The techniques used by Eilers and Katsura comes from the world of graph $C^{*}$ algebra, and so only leads to split short exact sequence. Their work leaves open the question of whether there is a non-split short exact sequence $0 \rightarrow A \rightarrow B \rightarrow \mathbb{C} \rightarrow 0$ with $A$ semiprojective and $B$ not semiprojective. In light of Eilers and Katsura's result we certainly expect such a sequence to exist, and indeed, as we shall see in Theorem 3 , it does.

This note is structured as follows: In Section 2 we prove two propositions that will be our main tools, in Section 3 we prove the main theorem.

\section{ToOlbox}

We will be working with pullbacks. Given two $*$-homomorphisms $\phi: A \rightarrow D$, $\psi: B \rightarrow D$, we write, by standard abuse of notation, the pullback of $A$ and $B$ taken over $\phi$ and $\psi$ as $A \oplus_{D} B$. That is $A \oplus_{D} B=\{(a, b) \in A \oplus B \mid \phi(a)=\psi(b)\}$. The pullback is universal for *-homomorphisms into $A$ and $B$ that agree after compositions with $\phi$ and $\psi$. For a detailed account of the theory of pullbacks (and pushouts) see [12].

Our first tool will let us produce new short exact sequences from old ones. In particular it gives us a way to alter a split short exact sequence to make it non-split.

Proposition 1. Suppose we are given two short exact sequence

$$
0 \rightarrow I \rightarrow A \stackrel{\pi}{\rightarrow} \mathbb{C} \rightarrow 0
$$

and

$$
0 \rightarrow J \rightarrow B \stackrel{\rho}{\rightarrow} \mathbb{C} \rightarrow 0 .
$$

Let $P$ be the pullback of $A$ and $B$ taken over $\pi$ and $\rho$. Then the following three sequences are short exact:

$$
\begin{aligned}
0 \rightarrow I \oplus J & \rightarrow P \\
0 & \rightarrow I \rightarrow \mathbb{C} \rightarrow 0, \\
0 & \rightarrow J \rightarrow P \rightarrow 0, \quad \text { and },
\end{aligned}
$$


Moreover (3) splits if and only if both (1) and (2) splits.

Proof. We begin by proving that (4) is exact. The map from $P$ to $A$ is simply projection onto the first coordinate, which is a surjection since both $\pi$ and $\rho$ are surjections. The kernel consists of pairs $(a, b) \in P$ with $a=0$, that is pairs $(0, b)$ where $\rho(b)=0$. Hence the kernel is $0 \oplus I \cong I$. A similar argument shows that (5) is exact.

We now consider (3). The map from $P$ to $\mathbb{C}$ takes a pair $(a, b)$ and sends it to $\pi(a)(=\rho(b))$. By the surjectivity of $\pi$ and $\rho$ we see that this is indeed a surjection. The kernel of this map is pairs $(a, b) \in P$ such that $\pi(a)=0=\rho(b)$, which is exactly $I \oplus J$.

The universal property of the pullback ensures that if (1) and (2) both split then (3) splits. On the other hand if we have a splitting from $\mathbb{C}$ to $P$, then simply composing that with the coordinate projections will show that (11) and (2) both split.

Remark 1. In the form of a diagram we have shown that if we are given sequences (1) and (2) as in the above proposition, then the following diagram commutes and has exact rows, columns and diagonal.

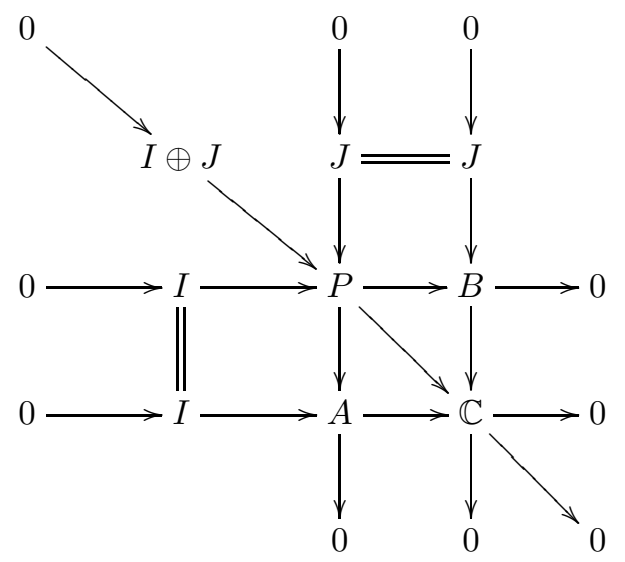

Now that we have a tool to construct non-split extensions from a split and a non-split one, we need a tool to tell us if the new extension is semiprojective. The following proposition is very slight generalization of [11, Proposition 5.19] (where the ideal has to be the stabilization of a unital $C^{*}$-algebra). The proofs are essentially identical, but since [1] is in German, we include a short proof.

Proposition 2. Consider a short exact sequence

$$
0 \rightarrow I \rightarrow A \stackrel{\rho}{\rightarrow} Q \rightarrow 0
$$

If $I$ is generated as an ideal by finitely many projections and $A$ is semiprojective then $Q$ is semiprojective.

Proof. Suppose we are given $B$, an increasing sequence of ideals $\left(J_{k}\right)$ in $B$, and a $*-$ homomorphism $\phi: Q \rightarrow B / J$, where $J=\overline{\cup_{k} J_{k}}$. For all $k \in \mathbb{N}$, we let $\pi_{k, \infty}: B / J_{k} \rightarrow$ $B / J$ be the natural quotient map. By the semiprojectivity of $A$ we can find and $n \in \mathbb{N}$ and a $*$-homomorphism $\psi: A \rightarrow B / J_{n}$ such that $\pi_{n, \infty} \circ \psi=\phi \circ \rho$.

Let $p_{1}, p_{2}, \ldots, p_{m}$ be projections that generate $I$. For all $i$ we have $\rho\left(p_{i}\right)=0$, and therefore we have $\left(\pi_{n, \infty} \circ \psi\right)\left(p_{i}\right)=0$. Hence, we can use [1, Lemma 2.13] to deduce that there must be some $l \geq n$ such that $\left(\pi_{n, l} \circ \psi\right)\left(p_{i}\right)=0$ for all $i=1,2, \ldots, m$. Since the $p_{i}$ generate $I$, we then have $\left(\pi_{m, k} \circ \psi\right)(I)=0$, so $\pi_{n, l} \circ \psi$ drops to a *-homomorphism $\bar{\psi}: Q \rightarrow B / J_{l}$ with $\pi_{l, \infty} \circ \bar{\psi}=\phi$. Thus $\bar{\psi}$ and $l$ combine to show that $Q$ is semiprojective. 
Our strategy is now the following: Find a non-split short exact sequence

$$
0 \rightarrow J \rightarrow B \rightarrow \mathbb{C} \rightarrow 0
$$

such that $J$ has a full projection. We will then use the construction in Proposition 1 on that and the Eilers-Katsura example, to produce a new non-split extension, which we can show, using Proposition 2] has the desired properties.

\section{Constructing a counterexample}

We begin this section by constructing a non-split short exact sequence where the ideal is semiprojective and contains a full projection, and the quotient is the complex numbers. To prove that the constructed sequence is non-split we will use $K$-theory. In particular, we will show that one of the boundary maps in the six-term exact sequence is non-zero. Since $K_{1}(\mathbb{C})=0$, we need a semiprojective $C^{*}$-algebra with non-zero $K_{1}$-group. We will use a Kirchberg algebra.

Definition 2. A separable, simple, nuclear, purely infinite $C^{*}$-algebras is called a Kirchberg algebra. If it also satisfies the universal coefficient theorem, we call it a UCT Kirchberg algebra.

Definition 3. Denote by $\mathcal{P}_{\infty}$ the unital UCT Kirchberg algebra with $K_{0}\left(\mathcal{P}_{\infty}\right)=0$ and $K_{1}\left(\mathcal{P}_{\infty}\right)=\mathbb{Z}$.

Building on the work of Blackadar ([2]) and Szymanski ([15]), Spielberg has shown in [14, Theorem 3.12] that any Kirchberg algebra with finitely generated $K$-theory and torsion free $K_{1}$-group is semiprojective. In particular we have:

Theorem 2 (Spielberg). Let $\mathbb{K}$ denote the algebra of compact operators. The Kirchberg algebra $\mathcal{P}_{\infty} \otimes \mathbb{K}$ is semiprojective.

We can now construct a non-split sequence with a semiprojective ideal that contains a full projection.

Proposition 3. There exists a non-split short exact sequence

$$
0 \rightarrow J \rightarrow E \rightarrow \mathbb{C} \rightarrow 0
$$

where $J$ is semiprojective and contains a full projection.

Proof. Put $J=\mathcal{P}_{\infty} \otimes \mathcal{K}$, as the stabilization of a unital algebra $J$ contains a full projection. By Theorem 2, it is semiprojective. We will pick $E$ such that the boundary map in $K$-theory from $K_{0}(\mathbb{C})$ to $K_{1}(J)$ is non-zero. Since $K$-theory is split exact this implies that the sequence does not split.

We have the following short exact sequence:

$$
0 \rightarrow J \rightarrow M(J) \rightarrow M(J) / J \rightarrow 0 .
$$

If we let $\eta: K_{0}(M(J) / J) \rightarrow K_{1}(J)$ be the boundary map in the six-term exact sequence arising from the above extension, then by [3, Proposition 12.2.1] $\eta$ is an isomorphism. In particular

$$
K_{0}(M(J) / J) \cong K_{1}(J) \cong K_{1}\left(\mathcal{P}_{\infty}\right)=\mathbb{Z} .
$$

By [8, Theorem 2.2], the corona algebra $M(J) / J$ has a continuous scale and so by [7. Theorem 3.2] it is simple and purely infinite. Since $M(J) / J$ is also unital there is, by [3. Corollary 6.11.8], a projection $p \in M(J) / J$ such that the class of $p$ in $K_{0}(M(J) / J)$ is $1 \in \mathbb{Z}$. Define a $*$-homomorphism $\tau: \mathbb{C} \rightarrow M(J) / J$ by $\tau(\lambda)=\lambda p$, and notice that $K_{0}(\tau)$ is an isomorphism of groups. 
Let $E=M(J) \oplus_{M(J) / J} \mathbb{C}$ where the pullback is taken over the quotient map from the multiplier algebra to the corona algebra and $\tau$. We have the following commutative diagram which has exact rows (see [17, Proposition 3.2.9]):

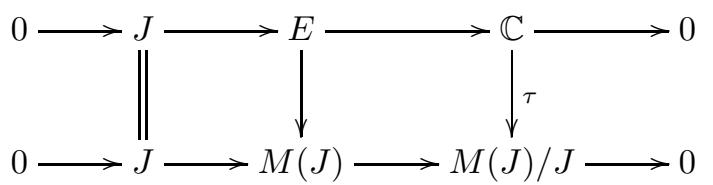

Let $\delta$ denote the boundary map from $K_{0}(\mathbb{C})$ to $K_{1}(J)$ in the six-term exact sequence associated to the short exact sequence on top. By [13, Proposition 12.2.1] the following square commutes:

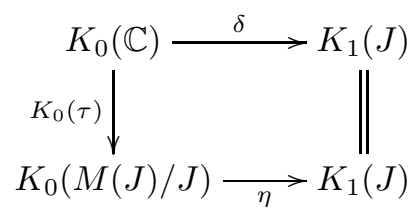

Since $\eta$ and $K_{0}(\tau)$ are isomorphisms, we must have that $\delta$ is an isomorphism. In particular $\delta$ is non-zero, so the sequence

$$
0 \rightarrow J \rightarrow E \rightarrow \mathbb{C} \rightarrow 0
$$

does not split.

We can now prove our main theorem.

Theorem 3. There exists a non-split short exact sequence

$$
0 \rightarrow K \rightarrow B \rightarrow \mathbb{C} \rightarrow 0,
$$

such that $K$ is semiprojective but $B$ is not.

Proof. Let

$$
0 \rightarrow I \rightarrow A \stackrel{\pi}{\rightarrow} \mathbb{C} \rightarrow 0
$$

be a short exact sequence such that $I$ is separable and semiprojective but $A$ is not semiprojective, e.g. one of the extensions constructed by Eilers and Katsura (Theorem 1), and let

$$
0 \rightarrow J \rightarrow E \stackrel{\rho}{\rightarrow} \mathbb{C} \rightarrow 0
$$

be the non-split extension constructed in Proposition 3 .

Put $B=A \oplus_{\mathbb{C}} E$ where the pullback is taken over $\pi$ and $\rho$. By Proposition 1 we have the following two short exact sequence:

$$
\begin{aligned}
0 \rightarrow I \oplus J & \rightarrow B \rightarrow \mathbb{C} \rightarrow 0, \quad \text { and }, \\
0 \rightarrow J & \rightarrow B \rightarrow A \rightarrow 0 .
\end{aligned}
$$

Furthermore (8) does not split as (6) does not split.

Since $J$ has a full projection and $A$ is not semiprojective Proposition2 2 applied to (8) gives us that $B$ is not semiprojective. To complete the proof we put $K=I \oplus J$ and notice that $K$ is semiprojective, as it is the sum of two separable semiprojective $C^{*}$-algebras ([9, Theorem 4.2]).

\section{Acknowledgments}

The author thanks Takeshi Katsura and Søren Eilers for bringing the problem to his attention. Søren Eilers is also thanked for giving discussions concerning the problem. This research was supported by the Danish National Research Foundation (DNRF) through the Centre for Symmetry and Deformation. 


\section{REFERENCES}

[1] Blackadaar, B.: Shape theory for $C^{*}$-algebras. Math. Scand. 56, $249-275$ (1985).

[2] Blackadar, B.: Semiprojectivity in simple $C^{*}$-algebras. In: Operator algebras and applications. Adv. Stud. Pure Math. textbf38, pp. 1-17. Math. Soc. Japan, Tokyo (2004)

[3] Blackadar, B.: $K$-theory for operator algebras. Cambridge University Press, Cambridge (1998).

[4] Eilers, S., Katsura, T.: Semiprojectivity and properly infinite projections in graph $C^{*}-$ algebras. In preparation.

[5] Eilers, S., Loring, T.A., Pedersen, G. K.: Stability of anticommutation relations: an application of noncommutative CW complexes. J. Reine Angew. Math. 499, 101 - 143 (1998).

[6] Enders, D.: Private communication (2011).

[7] Lin, H.: Simple corona $C^{*}$-algebras. Proc. Amer. Math. Soc. 132(11), 3215-3224 (2004).

[8] Lin, H., Zhang, S.: On infinite simple $C^{*}$-algebras. J. Funct. Anal. 100(1), 221-231 (1991).

[9] Loring, T. A.: Projective $C^{*}$-algebras. Math. Scand. 73, $274-280$ (1993).

[10] Loring, T. A.: Lifting solutions to perturbing problems in $C^{*}$-algebras. The American Mathematical Society, Providence (1997).

[11] Neubüser, B.: Semiprojektivität und Realisierungen von rein unendlichen $C^{*}$-Algebren. PhD Thesis, University of Münster (2000).

[12] Pedersen, G.K.: Pullback and pushout constructions in $C^{*}$-algebra theory. J. Funct. Anal. 167 243-344 (1999).

[13] Rørdam, M., Larsen, F., Laustsen, N.: An introduction to $K$-theory for $C^{*}$-algebras. Cambridge University Press, Cambridge (2000).

[14] Spielberg, J.: Semiprojectivity for certain purely infinite $C^{*}$-algebras. Trans. Amer. Math. Soc. 361(6), 2805-2830 (2009).

[15] Szymański, W.: On semiprojectivity of $C^{*}$-algebras of directed graphs. Proc. Amer. Math. Soc. 130, 1391-1399 (2002).

[16] Sørensen, A.P.W., Thiel, H.: A characterization of semiprojectivity for commutative $C^{*}$ algebras. Proc. London Math. Soc, to appear.

[17] Wegge-Olsen, N.E.: $K$-theory and $C^{*}$-algebras. Oxford University Press, Oxford (2004)

Department of Mathematical Sciences, University of Copenhagen, Universitetsparken

5, DK-2100, Copenhagen $\varnothing$, Denmark

E-mail address: apws@math.ku.dk 